\title{
Clima social familiar y autoconcepto en estudiantes de una institución educativa estatal
}

\section{Family social climate and self-concept in students of a state educational institution}

\author{
Edwin Estrada Araoz' ${ }^{1}$, Helen Mamani Uchasara²
}

\begin{abstract}
RESUMEN
Objetivo: determinar la relación que existe entre el clima social familiar y el autoconcepto en estudiantes de educación secundaria de una institución educativa estatal de El Triunfo. Metodología: el enfoque fue cuantitativo, el diseño no experimental y el nivel correlacional. La muestra fue conformada por 231 estudiantes del nivel de educación secundaria a través de un muestreo probabilístico estratificado a quienes se les aplicó la Escala de Clima Social Familiar (FES) de Moos y Trickett (1974) y la Escala AF-5 de García y Musitu (1999). Resultados: se halló mediante la prueba rho de Spearman que existe una correlación directa y significativa entre el clima social familiar y el autoconcepto $(r h o=0,532 ; p<0,05)$. Asimismo se halló correlación directa y significativa entre el clima social familiar y las dimensiones autoconcepto académico (rho $=0,459 ; p<0,05)$, autoconcepto social $($ rho $=0,343$; $p<0,05)$, autoconcepto emocional (rho $=0,552 ; p<0,05)$, autoconcepto familiar $(r h o=0,444 ; p<0,05)$ y autoconcepto físico (rho=0,559; $p<0,05$ ). Conclusión: los estudiantes que perciban un clima social familiar adecuado podrían tener un mejor autoconcepto.
\end{abstract}

Palabras clave: Clima social familiar, autoconcepto, adolescencia, educación secundaria

\begin{abstract}
Objective: to determine the relationship that exists between the family social climate and self-concept in secondary education students of a state educational institution in El Triunfo. Methodology: the approach was quantitative, the design was not experimental and the correlational level. The sample was made up of 231 students from the secondary education level through a stratified probabilistic sampling to which the Moos and Trickett (1974) Scale of Family Social Climate (FES) and the García y Musitu Scale AF-5 were applied. (1999). Results: it was found by Spearman's rho test that there is a direct and significant correlation between family social climate and self-concept (rho $=0.532 ; p$ $<0.05$ ). Likewise, a direct and significant correlation was found between the family social climate and the dimensions of academic self-concept ( $r h o=0.459 ; p<0.05$ ), social self-concept ( $r$ ho $=0.343 ; p$ $<0.05$ ), emotional self-concept (rho $=0.552 ; p<0.05$ ), family self-concept (rho $=0.444 ; p<0.05$ ) and physical self-concept (rho $=0.559 ; p<0.05$ ). Conclusion: students who perceive an adequate family social climate could have a better self-concept.
\end{abstract}

Keywords: Family social climate, self-concept, adolescence, secondary education.

\footnotetext{
${ }^{1}$ Universidad Nacional Amazónica de Madre de Dios, Madre de Dios, Perú Orcid ID: 0000-0003-4159-934X

2Universidad Nacional Amazónica de Madre de Dios, Madre de Dios, Perú Orcid ID: 0000-0003-1735-8570
} 


\section{INTRODUCCIÓN}

La dinámica familiar provoca cambios que se reflejan en los componentes cognitivos, afectivos y comportamentales de cada uno de sus integrantes (Isaza y Henao, 2011). En ese sentido, las familias que generen un ambiente caracterizado por una constante comunicación, expresividad, práctica de valores, organización de responsabilidades y cumplimiento de las normas, fomentarán en sus hijos un desarrollo adecuado de su persona (Guerrero y Mestanza, 2016). Por ello es necesario que el clima que se genere en la familia sea el más adecuado para favorecer el desarrollo integral, no solo de los hijos, sino de todos los integrantes.

De acuerdo a Moos y Trickett (1974) el clima social familiar es la percepción de las características socio-ambientales que presenta la familia, la cual se describe de acuerdo a interacción de los integrantes de la familia, los aspectos de desarrollo que son más relevantes en ella y su organización. En virtud a lo expuesto, las familias que tienen una organización cohesionada, realizan un control continuo y brindan afecto así como comprensión a sus hijos, permiten lograr un desempeño social continuo y consistente en ellos (Isaza y Henao, 2011).

El clima social familiar presenta 3 dimensiones: relaciones, desarrollo y estabilidad. La dimensión relaciones evalúa el nivel de comunicación y espontaneidad que se da en la familia y el nivel de interrelación conflictiva que la caracteriza. La dimensión desarrollo busca desarrollar los procesos de despliegue individual que ocurren y se fomentan dentro de la familia. Por último, la dimensión estabilidad está referida a la estructura y formación de la familia y a la manera cómo se ejerce el control entre sus integrantes (Santos, 2012).

Por otra parte, se ha evidenciado también que el entorno familiar también influye en la percepción que los hijos tienen de sí mismos, en todos los ámbitos en los que se relacionen (Malca y Rivera), es decir, fomenta un favorable autoconcepto. No obstante, la percepción de un clima familiar negativo repercutirá en cómo perciban la imagen de sí mismos, su autoestima y motivación (Romero, 2015).

El autoconcepto es definido por Salum, Marín y Reyes (2011) como "el conjunto de juicios tanto descriptivos como evaluativos acerca de uno mismo, es decir, en él se expresa el modo en que la persona se representa, conoce y valora a ella misma" (p.256). Bajo esa mirada, su función es fomentar que la persona se entienda, se guíe y pueda controlar o autorregular su conducta. Por otro lado García y Musitu (1999) señalan que el autoconcepto es entendido como la percepción que una persona tiene de sí misma, la cual se basa en sus experiencia con los otros y en las atribuciones de su propia conducta. En ese sentido, un autoconcepto adecuado promueve un correcto funcionamiento personal, social y profesional (Palacios y Coveñas, 2019).

García y Musitu (1999) realizó investigaciones sobre el autoconcepto indicando que presenta 5 dimensiones: el autoconcepto académico, social, emocional, familiar y físico. El autoconcepto académico se refiere a la sensación que la persona demuestra de la calidad de desarrollo de su propio papel, en el área que se desenvuelve. El autoconcepto social tiene que ver con la percepción que una persona tiene sobre su desenvolvimiento con los demás. En cuanto al autoconcepto emocional, se refiere a la forma cómo las personas ven su estado emocional y las respuestas a situaciones puntuales, con cierto nivel de responsabilidad e implicación en la vida cotidiana (García y Misuto, 1999). Respecto al autoconcepto familiar, tiene que ver con la percepción de las personas sobre cuán implicadas están en la participación e integración en el seno familiar. Finalmente, el autoconcepto físico se refiere a la forma cómo la persona ve y valora su aspecto y condición física.

De acuerdo a lo expuesto, la presente investigación tiene como objetivo general determinar la relación que existe entre el clima social familiar y el autoconcepto en estudiantes de educación secundaria de una institución educativa estatal de El Triunfo.

\section{METODOLOGÍA}

El enfoque de la presente investigación fue cuantitativo puesto que para realizar la prueba de hipótesis se procedió a recoger datos, lo cual hizo posible el análisis estadístico y la medición numérica de la información obtenida y sistematizada por los instrumentos que fueron aplicados (Sánchez, Reyes y Mejía, 2018). En cuanto al diseño, fue no experimental ya que las variables no fueron manipuladas 
deliberadamente, sino observadas tal como se dieron en su entorno y luego analizadas (Hernández, Fernández y Baptista, 2014). Asimismo, el nivel de investigación fue correlacional ya que se buscó conocer la relación que existe entre el clima social familiar y el autoconcepto (Bisquerra, 2009).

\section{Participantes}

La muestra fue conformada por un total de 231 estudiantes de ambos sexos, con edades que fluctuaban entre los 11 y 17 años y que cursaban del primero al quinto grado del nivel de educación secundaria de una institución educativa estatal de El Triunfo. Ellos fueron seleccionados mediante un muestreo probabilístico estratificado.

\section{Instrumentos}

Fueron utilizados 2 instrumentos de recojo de datos: la Escala de Clima Social Familiar (FES) y la Escala AF-5.

La Escala de Clima Social Familiar (FES) fue elaborada por Moos y Trickett (1974) y adaptada a la realidad peruana por Ruiz y Guerra (1993). Es utilizada para evaluar las características sociooambientales y las relaciones que se establecen dentro de la familia. Consta de 90 ítems estructurados en una escala dicotómica (verdadero y falso) y evalúan 3 dimensiones: relaciones, desarrollo y estabilidad. Para hallar las propiedades psicométricas de la escala se realizó el proceso de validez y confiabilidad. La validación de contenido fue realizada a través de la técnica de juicio de expertos, para lo cual se recurrió a 3 reconocidos especialistas en psicología. El promedio de las valoraciones fue de 0,80 lo cual indica que tiene una adecuada validez. En cuanto a la confiabilidad, a partir de una prueba piloto se obtuvo un coeficiente Kuder - Richardson (KR-20) de 0,820 lo que significa que el cuestionario tiene buena confiabilidad.

Con relación a Escala AF-5, fue elabor.-ada por García y Musitu (1999) y es usada para evaluar diferentes aspectos del autoconcepto en los niños, adolescentes y adultos. Presenta 30 ítems con una escala de tipo Likert (nunca, casi nunca, a veces, casi siempre y siempre) y evalúa 5 dimensiones: autoconcepto académico, social, emocional, familiar y físico. Para determinar las propiedades psicométricas de la escala se realizó el proceso de validez y confiabilidad. La validación de contenido también fue realizada mediante de la técnica de juicio de expertos obteniendo como promedio de las valoraciones 0,80 lo que significa que tiene una adecuada validez. Respecto a la confiabilidad, a partir de una prueba piloto se obtuvo un coeficiente Alfa de Cronbach de 0,844 lo que significa que el cuestionario también presenta buena confiabilidad.

\section{Procesamiento de datos}

Para realizar el análisis estadístico se recurrió al Software SPSS versión 22. Ahí los resultados fueron organizados y sistematizados en tablas de frecuencia y porcentaje. Para el análisis inferencial se utilizó la prueba no paramétrica rho de Spearman debido a la naturaleza de las variables y a que no se ajustan a la distribución normal $(p<0,05)$. Finalmente, para la significancia estadística, se consideró un valor menor o igual a 0,05 .

\section{RESULTADOS}

En la tabla 1 podemos observar que el $64,94 \%$ de los estudiantes manifiestan que el clima social familiar es regular, el $21,65 \%$ señala que es adecuado y el $13,42 \%$ precisa que es inadecuado. En cuanto a la dimensión relaciones, el $62,77 \%$ señala que es regular, el $19,05 \%$ indica que son inadecuadas y el $18,18 \%$ mencionan que son adecuadas. Respecto a la dimensión desarrollo, el $57,58 \%$ precisa que es regular, el 32,90\% menciona que es adecuado y el 9,52 señala que es inadecuado. Con relación a la dimensión estabilidad, el $82,68 \%$ de los estudiantes indican que es regular, el $12,12 \%$ señala que es inadecuada y el $5,19 \%$ menciona que es adecuada. Podemos observar de manera general que la mayoría de los estudiantes se caracterizan porque perciben que el ambiente familiar donde se desenvuelven no es muy favorable para su desarrollo cognitivo, afectivo y comportamental puesto que a veces se presentan conflictos, poca expresividad y disfuncionalidad lo cual genera regularmente el incumplimiento de las normas y disciplina en el hogar. 
Tabla 1

Niveles de clima social familiar y sus dimensiones

\begin{tabular}{lllllll}
\multirow{2}{*}{ Variable y dimensiones } & \multicolumn{2}{c}{ Inadecuado } & \multicolumn{3}{c}{ Regular } & \multicolumn{3}{c}{ Adecuado } \\
\cline { 2 - 7 } & $\mathrm{n}$ & $\%$ & $\mathrm{n}$ & $\%$ & $\mathrm{n}$ & $\%$ \\
Clima social familiar & 31 & 13,42 & 150 & 64,94 & 50 & 21,65 \\
Relaciones & 44 & 19,05 & 145 & 62,77 & 42 & 18,18 \\
Desarrollo & 22 & 9,52 & 133 & 57,58 & 76 & 32,90 \\
Estabilidad & 28 & 12,12 & 191 & 82,68 & 12 & 5,19 \\
\hline
\end{tabular}

En la tabla 2 podemos apreciar que el $64,5 \%$ de los estudiantes presentan un nivel de autoconcepto medio, el $25,11 \%$ presenta un nivel alto y el $10,39 \%$ un nivel bajo. Con relación al autoconcepto académico, el $60,61 \%$ tiene un nivel medio, el 20,78 presenta un nivel alto y el $18,61 \%$ tiene un nivel bajo. Respecto al autoconcepto social, el $77,06 \%$ de estudiantes tiene un nivel medio, el 11,69 presenta un nivel bajo y el $11,26 \%$ un nivel alto. En cuanto al autoconcepto emocional, el $71,86 \%$ presenta un nivel medio, el 19,48\% presenta un nivel alto y el 8,66 tiene un nivel bajo. Con relación al autoconcepto familiar, el $60,17 \%$ se encuentra en el nivel medio, el 22,51 presenta un nivel bajo y el $17,32 \%$ tiene un nivel alto. Finalmente, respecto al autoconcepto físico, el $53,68 \%$ de los estudiantes presenta un nivel medio, el $33,33 \%$ tiene un nivel bajo y el $12,99 \%$ presenta un nivel alto. De acuerdo a los resultados expuestos se puede indicar que la mayor parte de estudiantes se caracteriza porque tienen una opinión parcialmente adecuada se sí mismos, de sus actitudes, emociones y sobre su nivel de conocimiento de sus capacidades y aceptación social. Teniendo en cuenta que la adolescencia es la etapa donde el autoconcepto adquiere mayor importancia, es necesario consolidarlo para que se sientan seguros de sí mismos, se valoren y respeten.

Tabla 2

Niveles de autoconcepto y sus dimensiones

\begin{tabular}{|c|c|c|c|c|c|c|}
\hline \multirow{2}{*}{ Variable y dimensiones } & \multicolumn{2}{|c|}{ Bajo } & \multicolumn{2}{|c|}{ Medio } & \multicolumn{2}{|c|}{ Alto } \\
\hline & $\mathrm{n}$ & $\%$ & $\mathrm{n}$ & $\%$ & $n$ & $\%$ \\
\hline Autoconcepto general & 24 & 10,39 & 149 & 64,50 & 58 & 25,11 \\
\hline Autoconcepto académico & 43 & 18,61 & 140 & 60,61 & 48 & 20,78 \\
\hline Autoconcepto social & 27 & 11,69 & 178 & 77,06 & 26 & 11,26 \\
\hline Autoconcepto emocional & 20 & 8,66 & 166 & 71,86 & 45 & 19,48 \\
\hline Autoconcepto familiar & 52 & 22,51 & 139 & 60,17 & 40 & 17,32 \\
\hline Autoconcepto físico & 77 & 33,33 & 124 & 53,68 & 30 & 12,99 \\
\hline
\end{tabular}

De acuerdo a la información presentada en la tabla 3, el coeficiente de correlación rho de Spearman indica que existe una correlación moderada, directa y significativa entre el clima social familiar y el autoconcepto ( $r s=0,532 ; p=0,000<0,05$ ). Ello indica que si los estudiantes del nivel de educación secundaria perciben un clima social familiar adecuado podrán tener un mejor autoconcepto.

En cuanto a las dimensiones del autoconcepto, se puede ver que existe relación directa y significativa entre el clima social familiar y las dimensiones del autoconcepto. Esto significa que la percepción de un adecuado ambiente familiar está relacionado con altos niveles de autoconcepto académico ( $r s=0,459 ; p=0,000<0,05)$, autoconcepto social $(r s=0,343 ; p=0,000<0,05)$, autoconcepto emocional ( $r s=0,552 ; p=0,000<0,05)$, autoconcepto familiar $(r s=0,444 ; p=0,000<0,05)$ y autoconcepto físico (rs=0,559; $p=0,000<0,05)$. 
Tabla 3

Correlación entre el clima social familiar y el autoconcepto

\begin{tabular}{lll} 
& \multicolumn{2}{l}{ Clima social familiar } \\
\cline { 2 - 3 } Variable y dimensiones & rs & 0 \\
Autoconcepto general & $0,532^{* *}$ & 0,000 \\
Autoconcepto académico & $0,459^{* *}$ & 0,000 \\
Autoconcepto social & $0,343^{* *}$ & 0,000 \\
Autoconcepto emocional & $0,552^{* *}$ & 0,000 \\
Autoconcepto familiar & $0,444^{* *}$ & 0,000 \\
Autoconcepto físico & $0,559^{* *}$ & 0,000 \\
\hline
\end{tabular}

** La correlación es significativa al nivel 0.01

\section{DISCUSIÓN}

Un primer hallazgo en la presente investigación es que el $64,94 \%$ de los estudiantes manifiestan que el clima social familiar donde se desenvuelven es regular, el $21,65 \%$ señala que es adecuado y el $13,42 \%$ precisa que es inadecuado. Este resultado coincide con el hallazgo de Antón (2018) quien halló identificó que la mayoría de los estudiantes $(54,3 \%)$ percibía que el nivel clima social familiar era promedio lo cual significa que los miembros de la familia de los estudiantes en mención en ocasiones se encuentran compenetrados y se apoyan entre sí, la comunicación con los demás miembros de la familia es regular, muestran cierta tranquilidad y estabilidad en su comportamiento hacia ellos y a veces actúan libremente y expresan directamente sus sentimientos. Del mismo modo coinciden con el estudio de Cahuana y Carazas (2018) quienes hallaron que el 50.7\% de estudiantes presentaba un nivel de clima social familiar moderado, lo cual quiere decir que existe un grado relativo de comunicación, apoyo y control entre los miembros de la familia.

Respecto al autoconcepto, se pudo identificar que el $64,5 \%$ de los estudiantes presentan un nivel de autoconcepto medio, el $25,11 \%$ presenta un nivel alto y el $10,39 \%$ un nivel bajo. Estos resultados también corroboran los hallazgos de Antón (2018) quien determinó que el $31,4 \%$ de los estudiantes se ubican en el nivel normal de autoconcepto lo que significa que mantienen un adecuado concepto de sí mismos, tanto a nivel intelectual, personal, familiar y de aceptación social que le van a permitir un mejor afrontamiento de las situaciones sociales, ya que el estudiante al relacionarse con otros, muestra su forma de ser con los demás, y en la medida que el estudiante reciba la aceptación de su entorno social, se sentirá aceptado por la forma en que se perciba a sí mismo. También coincide con los resultados de Guerrero y Mestanza (2016) quienes determinaron en su estudio que los estudiantes presentan el $43.3 \%$ de los escolares presentan autoconcepto moderado, es decir, que los escolares de 14 a 16 años tienen una percepción parcialmente positiva de sí mismos y de las capacidades que poseen. Asimismo, guarda relación con Malca y Rivera (2019) quienes hallaron que el 50,3\% de los participantes presentaron un nivel medio de autoconcepto general, relacionado a su entorno familiar, su contexto académico, relaciones sociales, estado emocional y su aspecto físico.

En cuanto al contraste de hipótesis general, se determinó que existe una correlación moderada, directa y significativa entre el clima social familiar $y$ el autoconcepto ( $r s=0,532 ; p=0,000<0,05$ ). Ello indica que si los estudiantes viven en un ambiente donde las relaciones interpersonales son adecuadas y posibilitan su desarrollo, comunicación y crecimiento personal, su percepción y valoración sobre sí mismos será favorable. Este hallazgo guarda relación con la investigación de Malca y Rivera (2019) quienes establecieron que el clima social familiar y el autoconcepto se relacionaron significativamente en los adolescentes del estudio ( $r s=0,465$; $p=0,000<0,05$ ). Ello indica que el adolescente, al tener un ambiente familiar donde existe una buena comunicación, le dan importancia a los procesos de desarrollo personal y existe una adecuada estructura y organización, podrá desarrollarse en un ambiente que le permita tener un mejor autoconcepto. Asimismo, coincide con los hallazgos de Guerrero y Mestanza (2016) quienes demostraron que existe relación altamente significativa entre las el clima familiar y el autoconcepto, es decir, que cuando las 
familias de los estudiantes presentan un adecuado clima familiar, el autoconcepto de los mismos es favorable. Del mismo modo, guarda relación con los hallazgos de Antón (2018) quien determinó que existe relación significativa del clima social familiar y el autoconcepto en los estudiantes de segundo y tercer grado de secundaria de la I.E "José Gálvez Egusquiza” Talandracas-Provincia de Chulucanas, Piura 2016. En ese sentido, un clima social familiar positivo y constructivo fomenta el desarrollo emocionalmente estable de sus miembros. Por otro lado un clima familiar negativo con modelos inadecuados puede favorecer comportamientos desadaptados, falta de habilidades emocionales y carencias afectivas importantes.

Asimismo, se pudo determinar que existe una relación directa y significativa entre el clima social familiar y las dimensiones autoconcepto académico ( $r s=0,459 ; \quad p=0,000<0,05)$, autoconcepto social ( $r s=0,343 ; p=0,000<0,05$ ), autoconcepto emocional ( $r s=0,552$; $p=0,000<0,05$ ), autoconcepto familiar ( $r s=0,444$; $p=0,000<0,05)$ y autoconcepto físico ( $r s=0,559$; $p=0,000<0,05)$. Un clima familiar apropiado es caracterizado por la presencia de un ambiente que facilita el desarrollo y crecimiento de todos los integrantes que conforman el núcleo familiar, permitiendo su crecimiento y el logro de las tareas de desarrollo, de acuerdo con las etapas evolutivas.

En ese sentido, si un estudiante convive en una familia cuyas características sean similares a las mencionadas previamente, tendrá una adecuado juicio que le permitirá reconocerse y definirse favorablemente según sus dimensiones académico, social, emocional, familiar y físico. Este hallazgo coincide con los resultados reportados por Malca y Rivera (2019) quienes determinaron que el clima familiar se asocia de manera débil, directa y significativa $(p<0,05)$ a los factores del autoconcepto (autoconcepto académico, social, emocional, familiar y físico).

Finalmente, es necesario precisar que la presente investigación se limitó a indagar la relación entre el clima social familiar y el autoconcepto en una muestra de estudio. En ese sentido, no se compararon las diferencias en las variables analizadas según el género ni la edad. Se sugiere que a partir de los hallazgos encontrados, investigaciones futuras pueden indagar los aspectos referidos.
Declaración de financiamiento y de conflictos de interés:

El estudio fue financiado por los autores, quienes declaran no tener conflictos de interés

\section{Correspondencia}

Edwin Estrada Araoz

Correo electrónico:

edwin5721@outlook.com

María Horta Murcia

Correo electrónico:

reading.kvision@gmail.com

\section{REFERENCIAS BIBLIOGRÁFICAS}

Antón, S. (2018). Relación entre el clima social familiar y el autoconcepto de los estudiantes de segundo y tercer grado de secundaria de la I.E. "José Gálvez Egusquiza” Talandracas - provincia de Chulucanas, Piura 2016. (Tesis de Licenciatura, Universidad Católica los Ángeles de Chimbote), Recuperado de http://repositorio.uladech.edu.pe/ handle/123456789/5120

Bisquerra, R. (2009). Metodología de la Investigación Educativa. (2a ed.). Madrid: La Muralla S.A.

Cahuana, F. y Carazas, D. (2018). Clima Social Familiar y Resiliencia en adolescentes de 15 a 19 años de edad, internos del Centro Juvenil de Diagnóstico y Rehabilitación de Lima. (Tesis de Licenciatura, Universidad Peruana Unión) Recuperado de http://repositorio.upeu.edu.pe/ handle/UPEU/1009

García, F. y Musitu, G. (1999). AF5: Autoconcepto Forma 5. Madrid: TEA Ediciones.

Guerrero, S. y Mestanza, N. (2016). Clima Social Familiar y Autoconcepto en estudiantes de 3ro a 5to año de secundaria de una institución educativa privada de Lima este, 2015. (Tesis de Licenciatura, Universidad Peruana Unión) Recuperado de http://repositorio.upeu.edu.pe/ handle/UPEU/460

Hernández, R. Fernández, C. y Baptista, P. (2014) Metodología de la Investigación. ( $6^{\mathrm{a}}$ ed.). México: Mc Graw-Hill.

Isaza, L. y Henao, G. (2011). Relaciones entre el clima social familiar y el desempeño en habilidades sociales en niños y niñas entre dos y tres años de edad. Acta Colombiana de Psicología, 14 (1): 19-30. Recuperado de https:// 
actacolombianapsicologia.ucatolica.edu.co/ article/view/355

Malca, A. y Rivera, L. (2019). Clima social familiar ¿Qué relación tiene con el autoconcepto en adolescentes del Callao? CASUS. Revista De Investigación y casos en salud, 4(2), 120129. Recuperado de: https://doi.org/10.35626/ casus.2.2019.208

Moos, R. y Trickett, E. (1974). Classroom environment scale manual. California: Ed. Consulting Psychologist Press.

Palacios, J. y Coveñas J. (2019). Predominancia del autoconcepto en estudiantes con conductas antisociales del Callao. Propósitos y Representaciones, 7(2), 325-352. Recuperado de:http://dx.doi.org/10.20511/pyr2019.v7n2.278

Romero, C. (2015). El clima social familiar y el autoconcepto en los alumnos del 3ero de secundaria de la institución educativa "San Juan" de San Juan de Miraflores, 2015. (Tesis de Maestría,Universidad César Vallejo). Recuperado de http://repositorio.ucv.edu.pe/ handle/UCV/17962

Ruiz, C. y Guerra, E. (1993). Estandarización del TEST FES / "Escala de Clima Social en la familia para Lima Metropolitana".
Salum, A. Marín, A y Reyes, A. (2011). Relevancia de las dimensiones del autoconcepto en estudiantes de escuelas secundarias de Ciudad Victoria, Tamaulipas, México. Revista Electrónica de Psicología Iztacala, 14(2), 255-272. Recuperado de https://www.medigraphic.com/pdfs/ epsicologia/epi-2011/epi112n.pdf

Sánchez, H., Reyes, C. y Mejía, K. (2018). Manual de términos en investigación científica, tecnológica y humanística. Universidad Ricardo Palma: Lima.

Santos, L. (2012). El clima social familiar y las habilidades sociales de los alumnos de una institución educativa del Callao. (Tesis de Maestría Universidad San Ignacio de Loyola) Recuperado de http://repositorio.usil.edu.pe/ handle/123456789/1323

Recibido: 19/11/2019

Aceptado: 09/04/2020 